Check for updates

Cite this: RSC Adv., 2018, 8, 17551

\title{
Preparation of thermo-responsive drug-loaded nanofibrous films created by electrospinning
}

\begin{abstract}
Jianbo Li, (iD * Chengwei Peng, Zhimei Wang and Jie Ren (D)
We prepared thermosensitive and biocompatible drug-loaded nanofibrous films by an electrospinning technique using a block copolymer, poly( $N$-isopropylacrylamide)-b-poly(L-lactide) (PNLA), and poly(Llactide) (PLLA). The copolymer PNLA was synthesized by the radical polymerization of $N$ isopropylacrylamide (NIPAAm), followed by the ring-opening polymerization of L-lactide. The properties of PNIPAAm and PNLA were selectively discussed based on the results of NMR, FT-IR, GPC, and CA analyses. Because of the low molecular weight of PNIPAAm and PNLA and the hydrolysis of PNLA resulting from its hydrophilicity, these copolymers were inappropriate for electrospinning separately. Hence, a mixture of PNLA and PLLA was used to prepare electrospun nanofibrous films. SEM images of the PNLA/PLLA electrospun films showed that homogeneous fibres with smooth surfaces were obtained. In vitro release studies indicated that the drug-release rate of the PNLA/PLLA electrospun nanofibrous films can be adjusted by the content and molecular weight of PNLA and by the environmental temperature. The results demonstrate that electrospinning is a promising way to create stimuliresponsive fibrous films with potential applications in the design of controllable drug delivery systems.
\end{abstract}

Received 20th March 2018

Accepted 6th May 2018

DOI: $10.1039 / \mathrm{c} 8 \mathrm{ra02442a}$

rsc.li/rsc-advances techniques to modify PLA materials have been created in the past years. Among these techniques, an important method is to modify the electrospinning fabrication process and setup. For example, the structure and morphology of the fibre can be controlled by different parameters, ${ }^{14}$ such as the electric field strength, distance between the spinneret and the collecting plate, temperature and humidity, or by modification of the setup, including side-by-side electrospinning systems, ${ }^{22,23}$ multi-jet electrospinning ${ }^{24,25}$ and co-axial electrospinning. ${ }^{26}$ In addition to focusing on the parameters and setup, adjusting the material compositions also plays an important role in the improvement and refinement of electrospun fibres, allowing manipulation of the properties and structure. Among these modified formulations, using rational polymer material designs, including copolymers ${ }^{27}$ and polymer mixtures with organic $^{\mathbf{2 8 , 2 9}}$ and inorganic components, ${ }^{\mathbf{8}, 30-32}$ and adjusting the component ratio have been considered efficient schemes to generate desirable materials with hydrophilicity, ${ }^{28,33}$ antibacterial properties, ${ }^{34}$ biodegradability, ${ }^{33}$ spinning ability, ${ }^{35}$ and mechanical integrity. ${ }^{\mathbf{1 5}}$

In this study, we provided a new pathway incorporating biodegradable and thermosensitive poly( $N$-isopropylacrylamide) (generally abbreviated PNIPAAm) with PLLA as an electrospinning solution formulation to fabricate an excellent fibrous film that overcomes the previously reported deficiencies. PNIPAAm is a temperature-responsive polymer, the lower critical solution temperature (LCST) of which is at a temperature near that of the human body.
Institute of Nano and Biopolymeric Materials, School of Materials Science and Engineering, Key Laboratory of Advanced Civil Engineering Materials, Ministry of Education, Tongji University, 4800 Caoan Road, Shanghai 201804, China. E-mail: lijianbo@tongji.edu.cn; Fax: +86-21-33515906; Tel: +86-21-33515906 
In general, PNIPAAm-modified PLLA materials can be obtained through either copolymerization of PNIPAAm and PLA ${ }^{27}$ or mixtures of PNIPAAm and PLA ${ }^{36}$ (as we have previously reported). The problem is that, as reported by Dai et al. ${ }^{27}$ and $\mathrm{Hu}$ et al.,$^{37}$ the copolymer is difficult to electrospin into fibres when the parameters are changed. This could be attributed to the low molecular weight of PNIPAAm- $b$-PLLA. Additionally, the films electrospun from these mixtures did not show a desirable increase in the rate of drug release with an increase in temperature, which was ascribed to the blended, not encapsulated, drug-loaded models resulting from the incompatibility between PNIPAAm and PLA.

In this study, to avoid the problems mentioned above, PNIPAAm was added to PLA in the form of the copolymer PNIPAAm$b$-PLA and electrospun to produce a biodegradable and thermoresponsive fibrous film. PNIPAAm- $b$-PLA was synthesized by radical polymerization and ring-opening polymerization. Through blending with PLA, the electrospinnability of PNIPAAm- $b$-PLA improved, and the diameter of fibres can be well controlled by adjusting the proportion of PNIPAAm blocks in PNIPAAm- $b$-PLA and the amount of PLA in the mixture. The thermo-sensitivity of the fibrous films resulting from the $\mathrm{C}=\mathrm{O}$ and $\mathrm{N}-\mathrm{H}$ groups in the PNIPAAm blocks can also be manipulated by adjusting the proportion of PLA. The PLA/PNLA electrospun fibrous film prepared in this manner was used as a stimuli-responsive carrier of rifampicin in a drug-release system to prevent the formation of Gram-positive and Gramnegative microbial communities on prosthetic surfaces after major orthopaedic surgeries. ${ }^{38}$ A patient may undergo multiple surgeries and prolonged antibiotic treatment following the current standard of care. Thus, use of the prepared system in wound dressing would be of clinical significance.

\section{Experimental}

\subsection{Materials}

$N$-Isopropylacrylamide (NIPAAm), recrystallized twice with toluene/cyclohexane $(\mathrm{v} / \mathrm{v}, 40 / 60)$ solvents, was purchased from TCI (Tokyo). $N, N^{\prime}$-Azobisisobutyronitrile (AIBN), recrystallized twice with ethanol, was provided by Shanghai Chemical Reagent Co., Ltd. 2-Mercaptoethanol (98\%) and stannous octoate (96\%) were purchased from Alfa Aesar. L-Lactide was purified by recrystallization from toluene three times and dried in a vacuum at room temperature. Poly(L-lactide) (PLLA) with an average molecular weight of 180000 and dried in vacuum at $60{ }^{\circ} \mathrm{C}$ for $12 \mathrm{~h}$ was obtained from Shanghai TJL Co., Ltd. (China). Other reagents and solvents were purchased from Shanghai Chemical Reagent Co., Ltd. (China) and used without further processing.

\subsection{Synthesis of PNIPAAm}

PNIPAAm was synthesized via radical polymerization with 2mercaptoethanol as the chain-transfer agent and AIBN as the initiator. The typical detailed preparation procedures were as follows. NIPAAm (8.23 g/72.8 mmol), AIBN (6.9 mg/0.042 mmol, $0.06 \mathrm{~mol} \%$ ) and 2-mercaptopropanol (6.88 $\mathrm{mg}, 0.06 \mathrm{~mol} \%)$ were dissolved in DMF (30 mL) first, then nitrogen was bubbled through the solution for $30 \mathrm{~min}$ to remove oxygen from the solvent before polymerization, and the solution was polymerized at $70{ }^{\circ} \mathrm{C}$ for $20 \mathrm{~h}$ under nitrogen atmosphere. Afterward, the white solid was obtained through spin-evaporation condensing under reduced pressure, repeatedly precipitating three times in excess ether and suction filtering. Then, the product was washed twice with ether and dried in a vacuum oven for $24 \mathrm{~h}$. A white powder was finally obtained.

\subsection{Synthesis of PNIPAAm- $b$-PLLA(PNLA)}

With the terminal hydroxyl of PNIPAAm as the initiation point, PNLA was synthesized from L-lactide by ring-opening polymerization, and the synthesis route is shown in Scheme 1. The typical detailed preparation procedure was as follows. First, the macromolecule initiator PNIPAAm ( $1 \mathrm{~g}$ ) and L-lactide (5 g/34.7 $\mathrm{mmol})$ were added to dry xylene $(3 \mathrm{~mL})$, then the initiator stannous octoate $(0.1 \mathrm{~mol} \%$ of the monomer) was added after stirring to dissolve. Then, nitrogen was charged three times after evacuation to remove the air from the reaction flask, and the reactants were polymerized at $120{ }^{\circ} \mathrm{C}$ for $24 \mathrm{~h}$ afterward. Subsequently, the products were dissolved in a small amount of THF and then precipitated in excess diethyl ether three times. The white powder PNLA was finally obtained after suction filtering, washing twice with ethyl ether and drying in a vacuum oven.

\subsection{Nuclear magnetic resonance spectroscopy (NMR)}

Nuclear magnetic resonance (NMR) spectra of the samples were obtained on a Bruker DMX 500 NMR spectrometer operating at $400 \mathrm{MHz}$ using deuterated chloroform $\left(\mathrm{CDCl}_{3}\right)$ as solvent, and the chemical shifts were evaluated relative to tetramethylsilane (TMS).

\subsection{Attenuated total reflection Fourier-transform infrared (ATR FT-IR) spectra}

Attenuated total reflection Fourier-transform infrared (ATR FTIR) spectra of the powders, after being ground and compressed
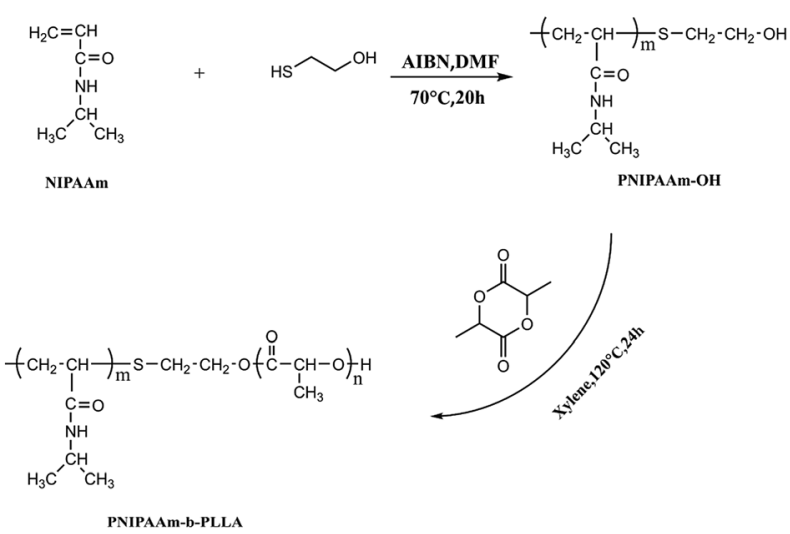

Scheme 1 Synthesis route of the amphiphilic block copolymer PNIPAAm- $b$-PLLA. 
with $\mathrm{KBr}$, were recorded on a magna FT-IR 550 FTIR spectrometer (Nicole, America) under the following parameter: measuring range $4000-400 \mathrm{~cm}^{-1}$.

\subsection{Gel permeation chromatography (GPC)}

The average molecular weight and polydispersity of the samples were measured on a gel permeation chromatograph instrument (GPC, WATERS-150C) at the flow rate of $1.0 \mathrm{~mL} \mathrm{~min}^{-1}$ using tetrahydrofuran (THF) as the eluent and polystyrene as the standard.

\subsection{Preparation of blank fibrous films}

The PLLA was dissolved in a mixed solvent composed of chloroform, dichloromethane and DMF (v/v/v: 7/2/1) first, and a concentration of $0.1 \mathrm{~g} \mathrm{~mL}^{-1}$ solution was obtained after magnetically stirring for $12 \mathrm{~h}$ at room temperature. Then, the solution was divided into two parts, one of which was used for electrostatic spinning directly, and the rest of the PLLA solution was added to PNLA powder slowly with further magnetic stirring for $12 \mathrm{~h}$ to formulate a solution of the desired concentration. Next, each of the obtained spinning solutions was transferred into a $20 \mathrm{~mL}$ syringe with a truncated-tip needle (9\#) for electrospinning, in which the spinning voltage was controlled at approximately $18 \mathrm{kV}$ and the receiving distance was $18-20 \mathrm{~cm}$, with the liquid feed rate adjusted by a micro injection pump at a flow rate of $2 \mathrm{~mL} \mathrm{~h}^{-1}$. After spinning for $4 \mathrm{~h}$, the film was dried in a vacuum at room temperature for one day. The dried fibrous membrane was finally peeled off from the aluminium foil and encapsulated in a self-sealing bag for further experimentation. For comparison, a casting film of PLLA was made on a Petri dish.

\subsection{Detecting the surface contact angle}

The contact angle was measured on an OCA20 contact-angle analysis system (DataPhysics, Germany). The temperature was controlled using a super thermostat (Julabo F25, Germany). Water droplets (approximately $2 \mu \mathrm{L}$ ) were dropped carefully onto the samples. The average CA values were obtained by measuring five different positions on the same sample.

\subsection{Preparation of drug-loaded fibres}

Preparation of the PLLA/PNLA solution was as described above. Rifampicin (RFP) powder was added to the solution such that an $8 \%$ drug loading (RFP vs. PLLA) was obtained. The procedures of electrospinning were also as described above.

\subsection{Scanning electron microscopy (SEM)}

The surface morphology of the fibrous films was investigated on a S-2360N scanning electron microscope (SEM, HITACHI, Japan) at a $15 \mathrm{kV}$ accelerating voltage after vacuum drying and gold plating. The average diameter and distribution of the ultrafine fibres in the SEM images were obtained by measuring one hundred fibres via Sigma Scan Pro4.0.

\subsection{In vitro release test}

PBS solution was used to simulate a human body fluid environment for the test of controlled drug-release ability. Fibrous films were cut into small pieces $(1 \mathrm{~cm} \times 1 \mathrm{~cm})$ and suspended into a wide jar containing PBS solution $(300 \mathrm{~mL}, \mathrm{pH} 7.4)$ that had been preheated to $25{ }^{\circ} \mathrm{C}$ or $40{ }^{\circ} \mathrm{C}$. The absorption peak at $\lambda_{\text {max }}=474 \pm 1 \mathrm{~nm}$ was measured by a UV-Vis spectrophotometer every other time. The cumulative release $\operatorname{Er}(\%)$ was calculated by the Lambert-Beer law.

\section{Results and discussion}

\subsection{Molecular structure of polymers}

The microstructure of the polymers was measured by FT-IR. Fig. 1 shows the spectrum of the homopolymer PNIPAAm. The $\mathrm{C}=\mathrm{O}$ stretching vibration peak and $\mathrm{N}-\mathrm{H}$ deformation vibration peak of the amide group are shown at $1655 \mathrm{~cm}^{-1}$ and $1544 \mathrm{~cm}^{-1}$, respectively. The peaks at $1386 \mathrm{~cm}^{-1}$ and $1367 \mathrm{~cm}^{-1}$ indicate the stretching vibration of the methyne groups on the pendant groups of PNIPAAm, and the peaks at $1332 \mathrm{~cm}^{-1}$ and $1272 \mathrm{~cm}^{-1}$ represent the doublet of overlapping $\mathrm{C}-\mathrm{N}$ and $\mathrm{C}-\mathrm{H}$. In addition, the $\mathrm{CH}_{3}$ scissor deformation $\left(1459 \mathrm{~cm}^{-1}\right), \mathrm{N}-\mathrm{C}$ stretching (1243 $\mathrm{cm}^{-1}$ and $\left.1172 \mathrm{~cm}^{-1}\right), \mathrm{C}-\mathrm{C}$ stretching $\left(1130 \mathrm{~cm}^{-1}\right), \mathrm{CH}_{3}$ rocking $\left(975 \mathrm{~cm}^{-1}\right.$ and $\left.925 \mathrm{~cm}^{-1}\right)$, and $\mathrm{CH}_{3}$ twisting $\left(883 \mathrm{~cm}^{-1}\right.$ and $\left.838 \mathrm{~cm}^{-1}\right)$ of the pendant groups are also presented in the spectrum. Moreover, disappearance of the $\mathrm{C}=\mathrm{C}$ stretching vibration peak at $1600 \mathrm{~cm}^{-1}$ indicated the acquisition of PNIPAAm. The FT-IR spectrum of the copolymer PNLA is also shown in Fig. 1. The peak at $1745 \mathrm{~cm}^{-1}$ indicates the $\mathrm{C}=\mathrm{O}$ stretching in the ester bond of PLLA, the $\mathrm{C}=\mathrm{O}$ stretching $\left(1655 \mathrm{~cm}^{-1}\right)$ and $\mathrm{N}-\mathrm{H}$ deformation $\left(1544 \mathrm{~cm}^{-1}\right)$ peaks, which are characteristic of PNIPAAm, did not disappear, supporting the conclusion that the copolymer PNLA was formed.

The molecular structure of the synthesized polymer was also analysed by nuclear magnetic resonance (NMR), and the spectrum is shown in Fig. 2. The spectrum of PNIPAAm (Fig. 2(a)) shows peaks at $\delta 4.0 \mathrm{ppm}$ and $\delta 1.2 \mathrm{ppm}$ that are attributed to the methyne and methyl protons of the $-\mathrm{NH}-\mathrm{CH}\left(\mathrm{CH}_{3}\right)_{2}$ groups in the NIPAAm moieties, respectively. The peaks at $\delta 1.5 \mathrm{ppm}$ and

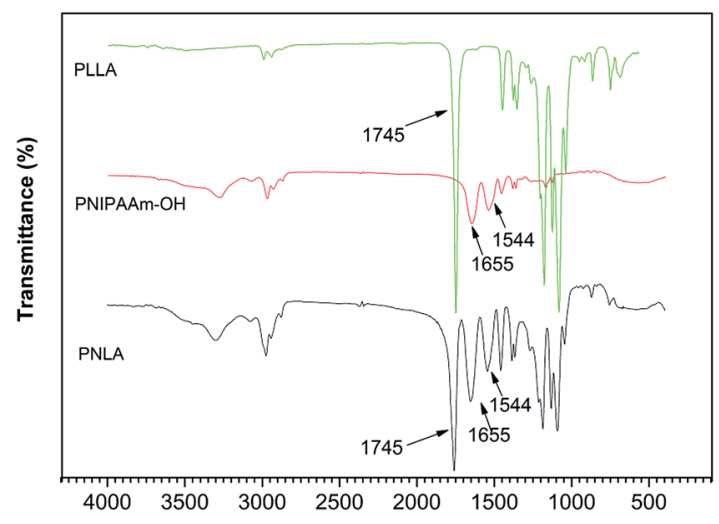

Fig. 1 FT-IR spectra of PLLA, PNIPAAm and PNLA. 


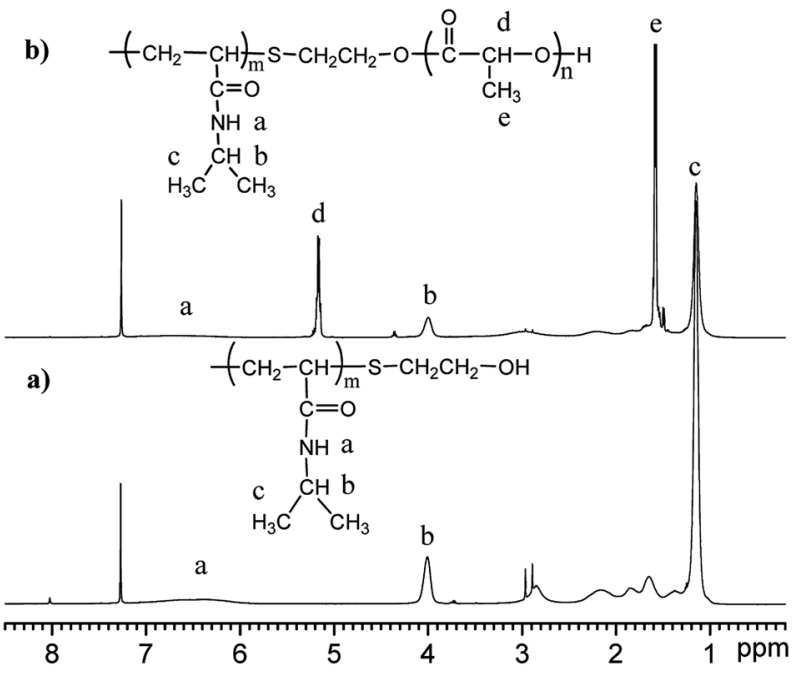

Fig. 2 The typical ${ }^{1} \mathrm{H}-\mathrm{NMR}$ spectra of (a) PNIPAAm and (b) PNLA in $\mathrm{CDCl}_{3}$.

$\delta 2.0 \mathrm{ppm}$ are attributed to the resonance of the protons of the methylene and methyne groups on the backbone of PNIPAAm, respectively. Since the hydrogen protons of $-\mathrm{NH}-\mathrm{CO}-$ are active and its resonance peak is generally not obvious, it's hard to identify without using heavy water exchange. The resonance peak is approximately assigned to the broad peak at $\delta 6.0-$ $7.0 \mathrm{ppm}$. The spectrum of PNLA is also shown in Fig. 2(b), in which the characteristic proton resonance peak $(\delta 1.6 \mathrm{ppm})$ of the methyne groups on the PLLA chain and the methyl peak $(\delta 5.2 \mathrm{ppm})$ on the side chain increased apparently. At the same time, the characteristic proton resonance peaks $(\delta 1.2 \mathrm{ppm}, \delta 4.0$ $\mathrm{ppm}$ ) of the double methyl and methyne groups on the pendant groups of the PNIPAAm chain can be seen clearly. Combining the results of FT-IR and ${ }^{1} \mathrm{HNMR}$, all of the signals confirmed the successful preparation of PNIPAAm and the copolymer PNLA.

\subsection{Molecular weight and distribution of polymers}

The molecular weights and distribution of the synthesized polymers are summarized in Table 1 . The $M_{\mathrm{n}}$ and $M_{\mathrm{w}} / M_{\mathrm{n}}$ values suggest that the molecular weights of both PNIPAAm and PNLA are too low to electrospin separately. ${ }^{39,40}$ Therefore, PNLA and PLLA were subsequently blended for electrospinning to obtain fibres with better structure.

\subsection{The surface contact angle of the fibrous films}

The wettability of the film was observed by water contact angle (CA) measurements. The coating film of pure PLLA [Fig. 3(a)]

Table 1 The characterization of PNIPAAm and PNLA block copolymers

\begin{tabular}{llll}
\hline Samples & PNIPAAm/LLA $(w t)$ & $M_{\mathrm{n}}$ & $M_{\mathrm{w}} / M_{\mathrm{n}}$ \\
\hline PNIPAAm & & 10500 & 1.65 \\
PNLA1 & $3: 10$ & 22600 & 1.59 \\
PNLA2 & $2: 10$ & 31600 & 1.31 \\
PNLA3 & $1: 10$ & 39700 & 1.45
\end{tabular}

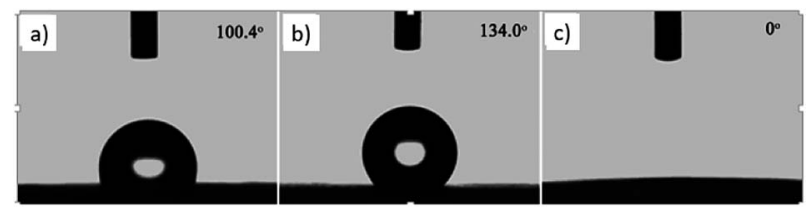

Fig. 3 Water contact angle of several samples: (a) casting film of PLLA, (b) electrospun film of PLLA, and (c) electrospun film of PNLA (bead-infibres).

shows a water contact angle of $100.4^{\circ}$, and the electrospun film of pure PLLA [Fig. 3(b)] shows a CA of $134^{\circ}$ at the same temperature $\left(20^{\circ} \mathrm{C}\right)$, which indicated an increase in the water contact angle resulting from the ultrafine fibrous film produced from the electrospinning process. The large water contact angle caused by strong hydrophobic interactions will slow down the release of drugs into aqueous fluids, enabling sustained release. A photograph of the water-droplet shape on the electrospun film of PNLA (bead-in-fibre) is shown in Fig. 3(c), which demonstrates that the suspended water droplets were quickly absorbed at $20{ }^{\circ} \mathrm{C}$ and that the water contact angle was nearly $0^{\circ}$. Obviously, the hydrophilicity of the PNLA electrospun film was caused by the PNIPAAm chain segments.

Table 2 shows the contact angles of the blend films with different proportions and at different temperatures. Combined with pure hydrophobic PLLA, the blended films showed more hydrophobicity. Among them, the PNLA1/PLLA $(3: 10)$ electrospun film showed the lowest contact angle of $120.5^{\circ}$ when the temperature was $25{ }^{\circ} \mathrm{C}$. Apparently, the contact angles of the PNLA/PLLA electrospun films were gradually enhanced with the increase in the hydrophobic PLLA proportion of the copolymer PNLA and the PNLA/PLLA blends. Additionally, the contact angles of the electrospun films also increased to some extent when the testing temperature increased from $25{ }^{\circ} \mathrm{C}$ to $40{ }^{\circ} \mathrm{C}$. This phenomenon was due to competition between the intermolecular and intramolecular hydrogen bonds of PNIPAAm as the temperature hovered around the LCST (approximately 32 $\left.{ }^{\circ} \mathrm{C}\right)$. When the temperature was lower than the LCST, despite the hydrophobic PLLA, the hydrophilic groups $\mathrm{N}-\mathrm{H}$ and $\mathrm{C}=\mathrm{O}$ of the amide bond on the PNIPAAm molecular chain tended to form intermolecular hydrogen bonds with the water molecules. Therefore, the PNLA/PLLA electrospun film was more hydrophilic. However, when the films were heated, intramolecular hydrogen bonds tended to form between the $\mathrm{C}=\mathrm{O}$ and $\mathrm{N}-\mathrm{H}$ groups, and the PNIPAAm molecular chain curled, which made it difficult for water molecules to interact with these hydrophilic groups. Therefore, the materials transformed to show more obvious hydrophobicity.

\subsection{Micro-morphology of the electrospun films}

As shown in Fig. 4(a) and (b), the morphology of the pure PNLA electrospun film was a microsphere structure instead of a fibre structure, which indicated that pure PNLA could not be electrospun into ultra-fine fibres. It is reasonable to speculate that the hydrophilicity of pure PNLA is excellent $\left(\mathrm{CA}=0^{\circ}\right.$ after spinning). In contrast, PLLA solution $(8 \% \mathrm{w} / \mathrm{v})$ could be spun 
Table 2 Water contact angles of films of different proportions at $25^{\circ} \mathrm{C}$ and $40{ }^{\circ} \mathrm{C}$

\begin{tabular}{llll}
\hline$T /\left({ }^{\circ} \mathrm{C}\right)$ & PNLA1 $:$ PLLA $=3: 10$ & PNLA2 : PLLA $=3: 10$ & PNLA1 : PLLA $=1: 10$ \\
\hline $120.5^{\circ}$ & $123.8^{\circ}$ & $126.1^{\circ}$ \\
$25^{\circ} \mathrm{C}$ & & \\
$40^{\circ} \mathrm{C}$ & &
\end{tabular}

into uniform fibres [Fig. 4(d)], and the fibres spun from PLLA solution $(10 \% \mathrm{w} / \mathrm{v})$ presented a "microbead/nanofibre" structure [Fig. 4(c)]. Obviously, PLLA has a great spinnability and will make up for the spinning deficiencies of PNLA. Therefore, combining PNLA with PLLA for blend electrospinning will provide better results to a certain extent.

\subsection{Micro-morphology and diameter distribution statistics of the drug-loaded PNLA/PLLA electrospun films}

Fig. 5 shows that the fibre diameter of PNLA/PLLA was very uniform and that there was no bead formation. Comparing Fig. 5(a) with 5(d), the average diameter of the ultra-fine fibres was proportional to the PNLA mass fraction, which was consistent with the previous analysis. Furthermore, with the increase in the molecular weight of PNLA, the average diameter of the fibres tended to decrease.
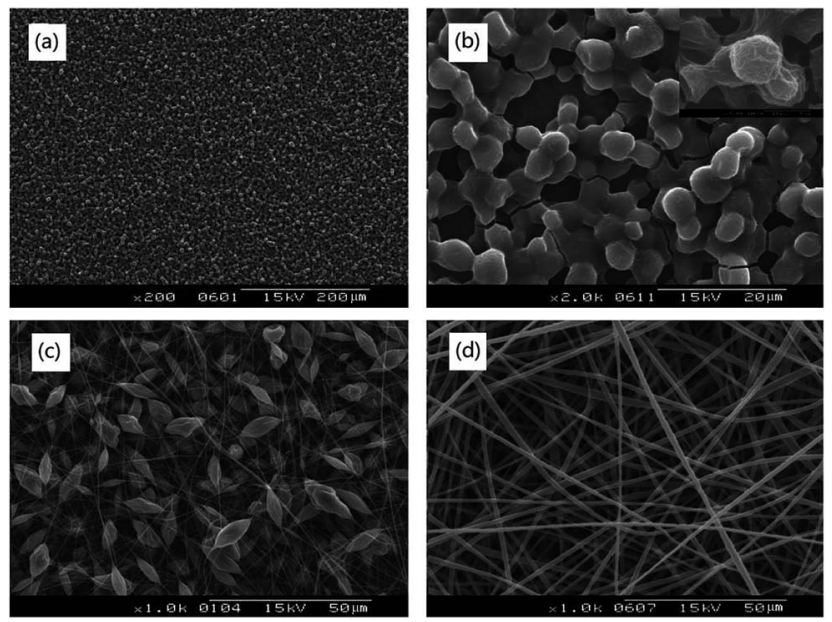

Fig. 4 SEM photographs of electrospun films: (a) and (b) PNIPAAm- $b$ PLLA(PNLA), (c) pure PLLA (10\%), (d) pure PLLA (8\%).
This effect was because as the molecular weight of PNLA increased, it became closer to the molecular weight of PLLA, and the spinning behaviour became much more similar to that
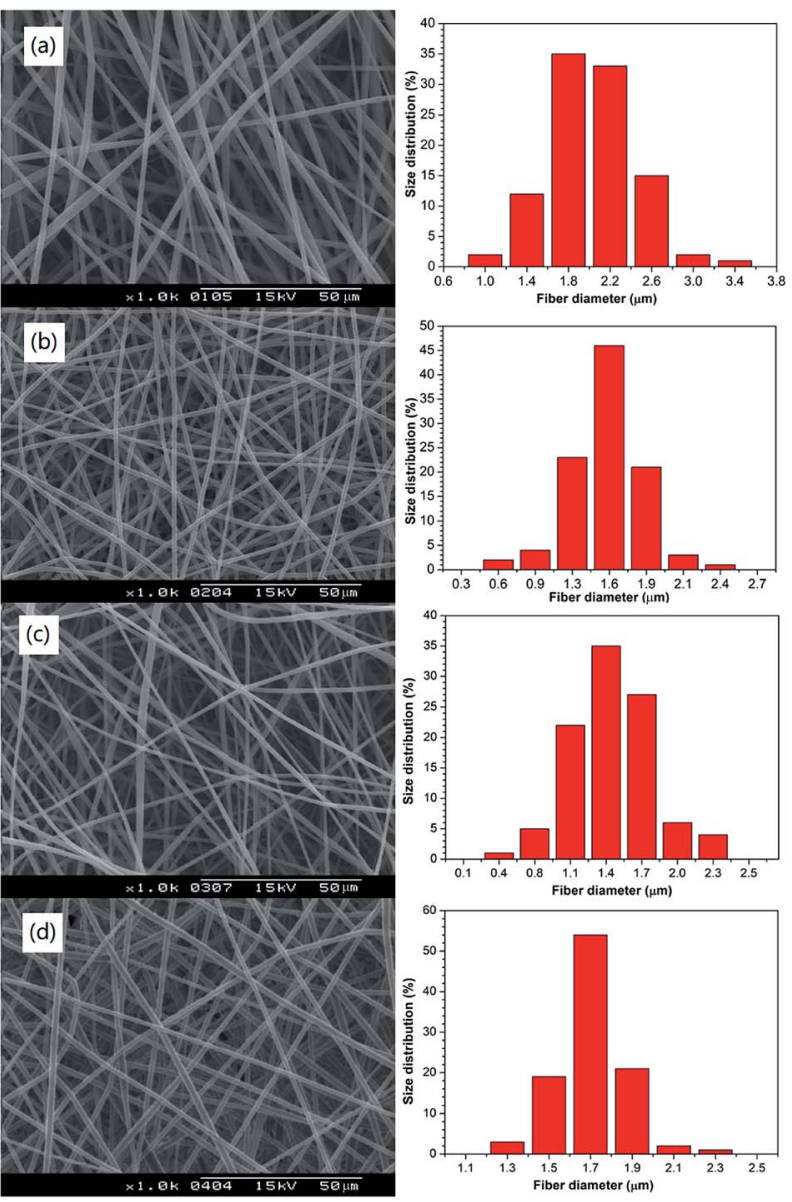

Fig. 5 SEM images and diameter distribution of the electrospun nanofibrous films of (a) PNLA1/PLLA $=3: 10$, (b) PNLA2/PLLA $=3: 10$, (c) PNLA3/PLLA $=3: 10$, and (d) PNLA1/PLLA $=1: 10$. 
Table 3 The average diameters of PNLA/PLLA drug-loaded nanofibres

\begin{tabular}{lll}
\hline & & $\begin{array}{l}\text { Average fibre } \\
\text { diameter }(\mu \mathrm{m})\end{array}$ \\
\hline $\mathrm{a} \#$ & PNLA1 $(22$ 600)/PLLA $=3: 10$ & 1.95 \\
$\mathrm{b \#}$ & PNLA2 $(31$ 600)/PLLA $=3: 10$ & 1.57 \\
$\mathrm{c \#}$ & PNLA3 (39 700)/PLLA $=3: 10$ & 1.49 \\
$\mathrm{~d} \#$ & PNLA1 $(22600) /$ PLLA $=1: 10$ & 1.73
\end{tabular}

of PLLA. Table 3 shows the average fibre diameters of PNLA/ PLLA films (a to d), and the results are in line with Fig. 5.

\subsection{In vitro drug-release behaviour of the drug-loaded PNLA/PLLA electrospun nanofibrous films}

The drug-release behaviour of the drug-loaded electrospun nanofibrous films was studied using a hydrophobic drug that was physically loaded and stabilized in the ultra-fine fibres of PNLA/PLLA electrospun films. Rifampicin (RFP), an antipneumonia drug with very low solubility in water, was chosen
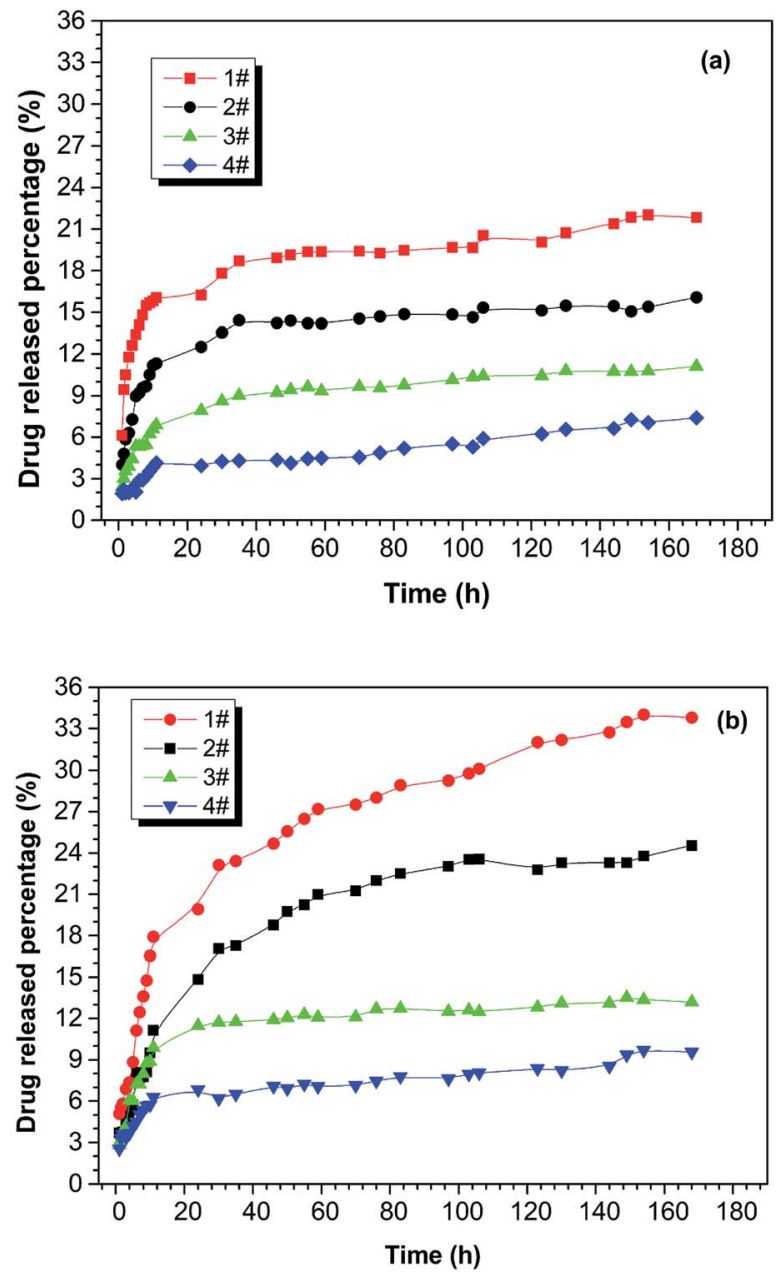

Fig. 6 Drug release curves of the drug-loaded electrospun nanofibrous films of $1 \#$ PNLA1/PLLA $=3: 10,2 \#$ PNLA2/PLLA $=3: 10,3 \#$ PNLA3/PLLA $=3: 10,4 \#$ PNLA1/PLLA $=1: 10$ at (a) $25{ }^{\circ} \mathrm{C}$ and (b) $40{ }^{\circ} \mathrm{C}$. as the model drug. Fig. 6 demonstrates the in vitro release curves of drug-loaded PNLA/PLLA electrospun films at different temperatures. By comparing Fig. 6(a) and (b), it can be seen that the electrospun nanofibrous films had higher drug release rates at $40{ }^{\circ} \mathrm{C}$ than at $25{ }^{\circ} \mathrm{C}$ which indicated that the prepared electrospun films possessed thermosensitive drug-release behaviour. This was because at higher temperature, PNIPAAm tends to form intramolecular hydrogen bonds, causing curling of the molecular chains and release of the contained drug. ${ }^{41,42}$ From the $1 \#, 2 \#$, and $3 \#$ curves in Fig. 6 , it could be found that the drug release rate of the electrospun films became significantly faster at the same temperature with an increase in the molecular weight of PNLA. The copolymer PNLA with the lower molecular weight contains more PNIPAAm structural units, which could lead to more thermosensitive amide bonds in electrospun nanofibrous films. Additionally, the drug release rate of the PNLA1/PLLA $(3: 10)$ electrospun film was dramatically higher than that of the PNLA1/PLLA $(1: 10)$ electrospun film. The significant increase in the drug release rate is believed to be attributable to the blending of more PNIPAAm chains into the PNLA/PLLA electrospun films. Therefore, the drug-release rate of the PNLA/PLLA electrospun nanofibrous films can be adjusted by the content and molecular weight of PNLA and by the environmental temperature.

\section{Conclusions}

Thermosensitive and biocompatible drug-loaded nanofibrous films were produced by an electrospinning technique using a PNLA/PLLA blend. The copolymer PNLA was synthesized by the radical polymerization of $N$-isopropylacrylamide, followed by the ring-opening polymerization of L-lactide. The molecular structures of PNIPAAm and PNLA were verified by the results of NMR, FT-IR, GPC, and CA analyses. Because of the low molecular weight and hydrophilicity of PNIPAAm and PNLA, they are unsuitable for application in electrospinning. Hence, a mixture of PNLA and PLLA was used to prepare electrospun nanofibrous films with different morphologies and diameters. Finally, the results indicated that the drug-release rate of the PNLA/PLLA electrospun nanofibrous films could be adjusted by the content and molecular weight of PNLA and by the environmental temperature. The results demonstrate that electrospinning is a promising way to create stimuli-responsive fibrous films with potential applications in the design and application of controllable drug delivery systems.

\section{Conflicts of interest}

There are no conflicts to declare.

\section{Acknowledgements}

This work was financially supported by the National High-Tech R\&D Program of China (No. 2013AA032202), the National Natural Science Foundation of China (No. 51203118), the Fundamental Research Funds for the Central Universities and the Open Funds for Characterization of Tongji University. 


\section{Notes and references}

1 F. Anton, US pat., 1,975,504, 1934.

2 J. M. Deitzel, J. D. Kleinmeyer, J. K. Hirvonen and N. C. B. Tan, Polymer, 2001, 42, 8163-8170.

3 P. Ke, X.-N. Jiao, X.-H. Ge, W.-M. Xiao and B. Yu, $R S C A d v$, 2014, 4, 39704-39724.

4 Y.-L. Cheng, J.-T. Chen, X.-B. Yan, Z.-M. Zheng and Q.-J. Xue, RSC Adv., 2013, 3, 20606-20612.

5 S. S. Ray, S.-S. Chen, C.-W. Li, N. C. Nguyen and H. T. Nguyen, RSC Adv., 2016, 6, 85495-85514.

6 C.-Q. Chen, Y. Yu, W. Li, C.-Y. Cao, P. Li, Z.-F. Dou and W. G. Song, J. Mater. Chem., 2011, 21, 12836-12841.

7 W.-J. Ma, Q.-L. Zhang, D.-W. Hua, R.-H. Xiong, J.-T. Zhao, W.-D. Rao, S.-L. Huang, X.-X. Zhan, F. Chen and C.-B. Huang, RSC Adv., 2016, 6, 12868-12884.

8 S.-J. Shao, S.-B. Zhou, L. Li, J.-R. Li, C. Luo, J.-X. Wang, X.-H. Li and J. Weng, Biomaterials, 2011, 32, 2821-2833.

9 F. Mohabatpour, A. Karkhaneh and A. M. Sharifi, RSC Adv., 2016, 6, 83135-83145.

10 J. Zhang, H. Liu, J.-X. Ding, X.-L. Zhuang, X.-S. Chen and Z.-M. Li, RSC Adv., 2015, 5, 32604-32608.

11 L.-L. Wu, X.-Y. Yuan and J. Sheng, J. Membr. Sci., 2005, 250, 167-173.

12 X.-H. Ma, S.-W. Gu and Z.-L. Xu, Catal. Today, 2016, 276, 133-138.

13 C.-Q. Chen, W. Li, C.-Y. Cao and W.-G. Song, J. Mater. Chem., 2010, 20, 6968-6974.

14 L.-H. Zhang, X.-P. Duan, X. Yan, M. Yu, X. Ning, Y. Zhao and Y.-Z. Long, RSC Adv., 2016, 6, 53400-53414.

15 C. M. Vaz, S. V. Tuijl, C. V. C. Bouten and F. P. Baaijens, Acta Biomater., 2005, 1, 575-582.

16 J.-W. Qin, Y.-Y. Jiang, J.-J. Fu, Y.-Q. Wan, R.-H. Yang, W.-D. Gao and H.-B. Wang, Iran. Polym. J., 2013, 22, 729-737.

17 A. Balaji, M. V. Vellayappan, A. A. John, A. P. Subramanian, S. K. Jaganathan, E. Supriyanto and S. I. A. Razak, $R S C$ Adv., 2015, 5, 57984-58004.

18 M. V. Vellayappan, J. R. Venugopal, S. Ramakrishna, S. Ray, A. F. Ismail, M. Mandal, A. Manikandan, S. Seal and S. K. Jaganathan, RSC Adv., 2016, 6, 83638-83655.

19 C.-T. Yeh and C.-Y. Chen, RSC Adv., 2017, 7, 6023-6030.

20 J. Ren, Biodegradable Poly (Lactic Acid): Synthesis, Modification, Processing and Applications, Springer Press, 2011.

21 T. Yu, J. Ren, S.-M. Li, H. Yuan and Y. Li, Composites, Part A, 2010, 41, 499-505.
22 H. Nie, B. W. Soh, Y.-C. Fu and C.-H. Wang, Biotechnol. Bioeng., 2008, 99, 223-234.

23 C. L. Li, C. Liu, W. Wang, J. Bell, Z. Mutlu, K. Ahmed, R. Ye, M. Ozkan and C. S. Ozkan, Chem. Commun., 2016, 52, 1139811401.

24 W.-G. Cui, X.-H. Li, S.-B. Zhou and J. Weng, J. Biomed. Mater. Res., Part A, 2007, 82, 831-841.

25 W. Li, C.-Y. Cao, C.-Q. Chen, Y. Zhao, W.-G. Song and L. Jiang, Chem. Commun., 2011, 47, 3619-3621.

26 B. Sun, B. Duan and X.-Y. Yuan, J. Appl. Polym. Sci., 2006, 102, 39-45.

27 X.-H. Dai, H. Jin, M.-H. Cai, H. Wang, Z.-P. Zhou, J.-M. Pan, X.-H. Wang, Y.-S. Yan, D.-M. Liu and L. Sun, React. Funct. Polym., 2015, 89, 9-17.

28 X.-Q. Shan, F.-Q. Li, C.-S. Liu and Q. Gao, J. Appl. Polym. Sci., 2014, 131, 41098.

29 P. Sarazin, X. Roy and B. D. Favis, Biomaterials, 2004, 25, 5965-5978.

30 Z.-Q. Su, J.-W. Ding and G. Wei, RSC Adv., 2014, 4, 5259852610.

31 A. Lazaro, S. Haddad, S. Sacca, C. Orellana-Tavra, D. FairenJimenez and R. S. Forgan, Chem, 2017, 2, 561-578.

32 M. S. Kwon, J. H. Jordahl, A. W. Phillips, K. Chung, S. Lee, J. Gierschner, J. Lahann and J. Kim, Chem. Sci., 2016, 7, 2359-2363.

33 E.-R. Kenawy, F. I. Abdel-Hay, M. H. El-Newehy and G. E. Wnek, Mater. Sci. Eng., A, 2007, 459, 390-396.

34 X.-Y. Xu, Q.-B. Yang, Y.-Z. Wang, H.-J. Yu, X.-S. Chen and X.-B. Jing, Eur. Polym. J., 2006, 42, 2081-2087.

35 W. Tomaszewski, W. Swieszkowski, M. Szadkowski, M. Kudra and D. Ciechanska, J. Appl. Polym. Sci., 2012, 125, 4261-4266.

36 S.-Y. Gu, Z.-M. Wang, J.-B. Li and J. Ren, Macromol. Mater. Eng., 2010, 295, 32-36.

37 Y.-F. Hu, V. Darcos, S. Monge and S.-M. Li, J. Polym. Sci., Part A: Polym. Chem., 2013, 51, 3274-3283.

38 H. V. D. Belt, D. Neut, W. Schenk, J. R. V. Horn, H. C. V. D. Mei and H. J. Busscher, Acta Orthop. Scand., 2001, 72, 557-571.

39 H. Lee, H. Y. Kim, H. J. Bang, Y. H. Jung and S. G. Lee, Polymer, 2003, 44, 4029-4034.

40 K. T. Tan and S. K. Obendorf, J. Membr. Sci., 2007, 305, 287298.

41 C.-L. Lo, K.-M. Lin and G.-H. Hsiue, J. Controlled Release, 2005, 104, 477-488.

42 C.-Y. Choi, S. Y. Chae and J.-W. Nah, Polymer, 2006, 47, 45714580. 